\title{
FIB-SEM Tomography and Its Application Towards Understanding Aerosol Optical Properties of Atmospheric Dust Particles
}

\author{
Diana Ortiz-Montalvo ${ }^{1}$, Robert Willis ${ }^{2}$ and Joseph Conny ${ }^{1}$ \\ ${ }^{1}$ National Institute of Standards and Technology, Gaithersburg, Maryland, United States, ${ }^{2}$ U.S. \\ Environmental Protection Agency (retired), Chapel Hill, North Carolina, United States
}

Atmospheric dust particles have complex morphologies as observed in scanning electron microscopy (SEM) images. Yet, despite their non-spherical shape and the growing evidence of shape-related uncertainty, these particles are commonly treated as spheres or spheroids in aerosol radiative forcing models and aerosol optical remote sensing applications [1,2]. Dust particles are known to have a strong influence on Earth's radiative balance. However, the extent to which they cool or warm the Earth's atmosphere remains largely uncertain [3]. Aerosol optical properties influence such cooling or warming behavior to a large extent, which depends on particle morphology, composition, size, and other factors. Our work aims to improve current understanding of aerosol optical properties by using high-resolution information on the shapes, volumes and surface roughness of ambient dust particles as obtained from focused ion-beam (FIB) SEM tomography, as well as elemental composition from energy-dispersive xray spectroscopy (EDS).

This study uses FIB-SEM (FEI Nova NanoLab 600 DualBeam) [4] equipped with a gallium ion source, Schottky field-emission gun, and Oxford silicon drift detector to perform serial sectioning and elemental mapping of individual ambient dust particles collected from urban and Asian sources. The complex threedimensional (3D) structures of the particles were generated by stacking the FIB-SEM images of each slice and doing image segmentation with FEI Avizo 7.1 Software. EDS elemental maps were used to identify different chemical phases and assign a complex refractive index to each material. Figure 1 shows an example of how different datasets were combined to generate the 3D model of the particles. The main benefit of applying FIB-SEM tomography to atmospheric particles is that the 3D models depict the particles' exact size, shape, and surface roughness, which are key in understanding their optical properties. To calculate particle optical properties, the coordinates of the cubic voxels that comprise the 3D reconstructions were exported from Avizo and then imported into a discrete dipole approximation (DDA) code (DDSCAT ver 7.3) [5]. DDA is open-source software that calculates scattering and absorption of electromagnetic waves by targets with arbitrary geometries and complex refractive indexes, making it suitable for calculating a variety of geometries and chemical compositions like those of ambient dust particles. Optical properties where calculated for the complex 3D model shape of the particles, as well as for (theoretical) volume-equivalent geometrical shapes like spheres, cubes, and tetrahedrons.

Overall, our studies using FIB-SEM tomography and optical property modeling have shown that the shape of a particle plays a vital role in its light-scattering properties and that using spherical or spheroidal shape models can generate large deviations in those properties. Furthermore, that optical property estimates from tetrahedral shapes had better agreement with the actual particle shape, when compared to spherical and cubic shapes. In Conny \& Ortiz-Montalvo (2017) we performed FIB-SEM tomography on heterogenous urban dust particles collected at Seattle, WA, Los Angeles, CA and Atlanta, GA [6]. We demonstrated that the shape was the dominating factor when evaluating variations in the light extinction efficiency and asymmetry parameter between the complex 3D particle shapes and equivalent geometric shapes. In most cases, shape was even more important than composition heterogeneity. Also, of the geometric shapes considered, the tetrahedron was the closest to match the extinction efficiency obtained for the complex 3D structures. More recently, we performed FIB-SEM tomography on heterogeneous dust particles 
collected at Hawaii's Mauna Loa Observatory during an Asian dust incursion event [7]. Our latest work uses those 3D models to understand how shape and surface roughness affect their optical properties [8]. Once again, we found that shape plays an important role in light scattering properties. In this work we used a surface smoothing algorithm in Avizo to vary the surface roughness of the 3D particle models without significantly changing the volume of the particle. Figure 2 shows a schematic of the trends observed as surface roughness increases, where light extinction increases but the backscatter fraction decreases. Finally, we compared the extinction efficiency and backscatter fraction of a series of geometrical shapes representing a progression in shape complexity and varying angularity. Of the 9 variations tested, the tetrahedron and triangular pyramid performed the best. Both shapes have high angularity and therefore more surface area that could account for scattering due to surface roughness in dust particles. Based on our findings we recommend the use of tetrahedral shape models in remote sensing applications and radiative forcing calculations to best represent the shape irregularity and surface roughness of ambient dust particles.

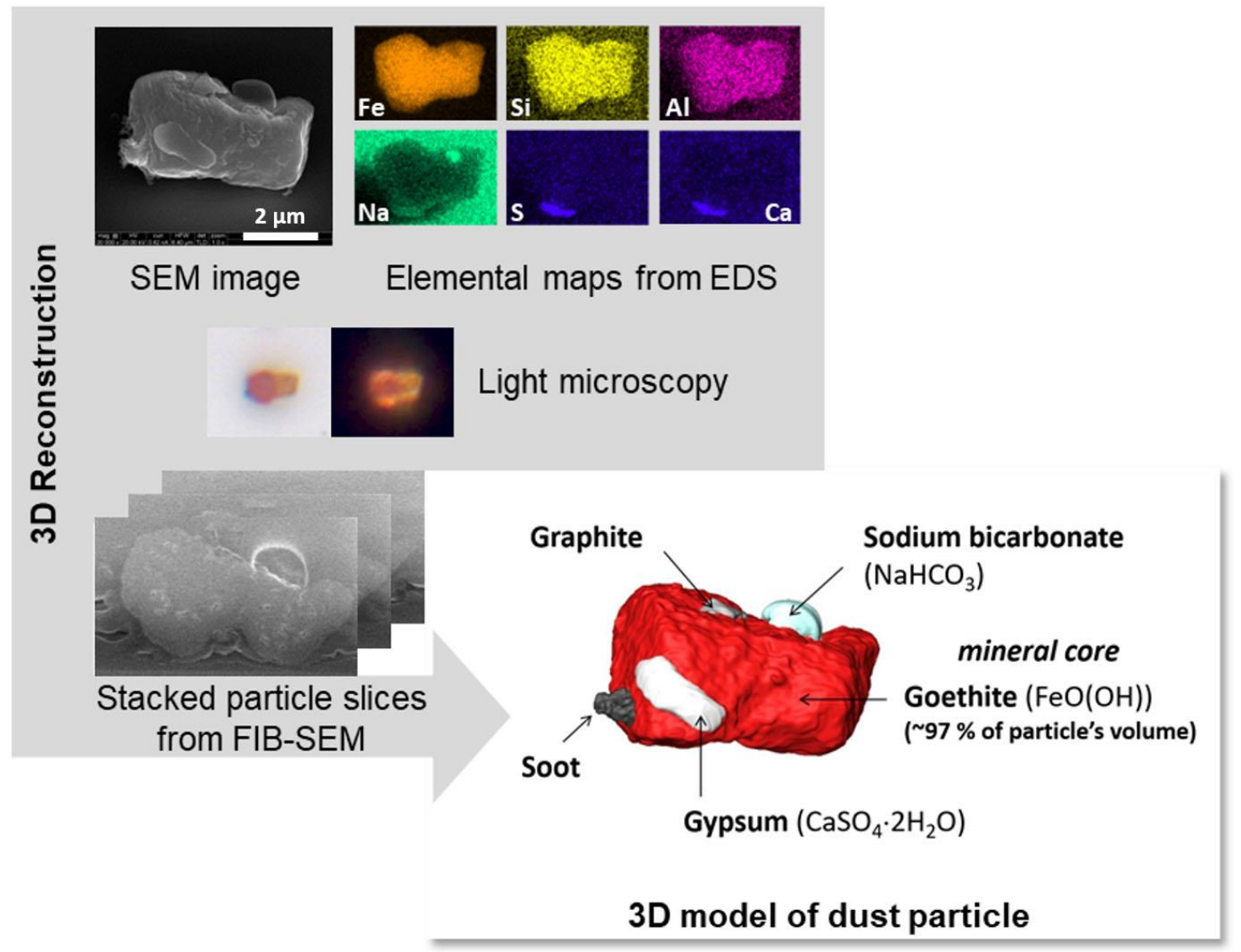

Figure 1. Diagram of the data used to make the 3D reconstruction of a representative dust particle collected at Seattle, WA. 


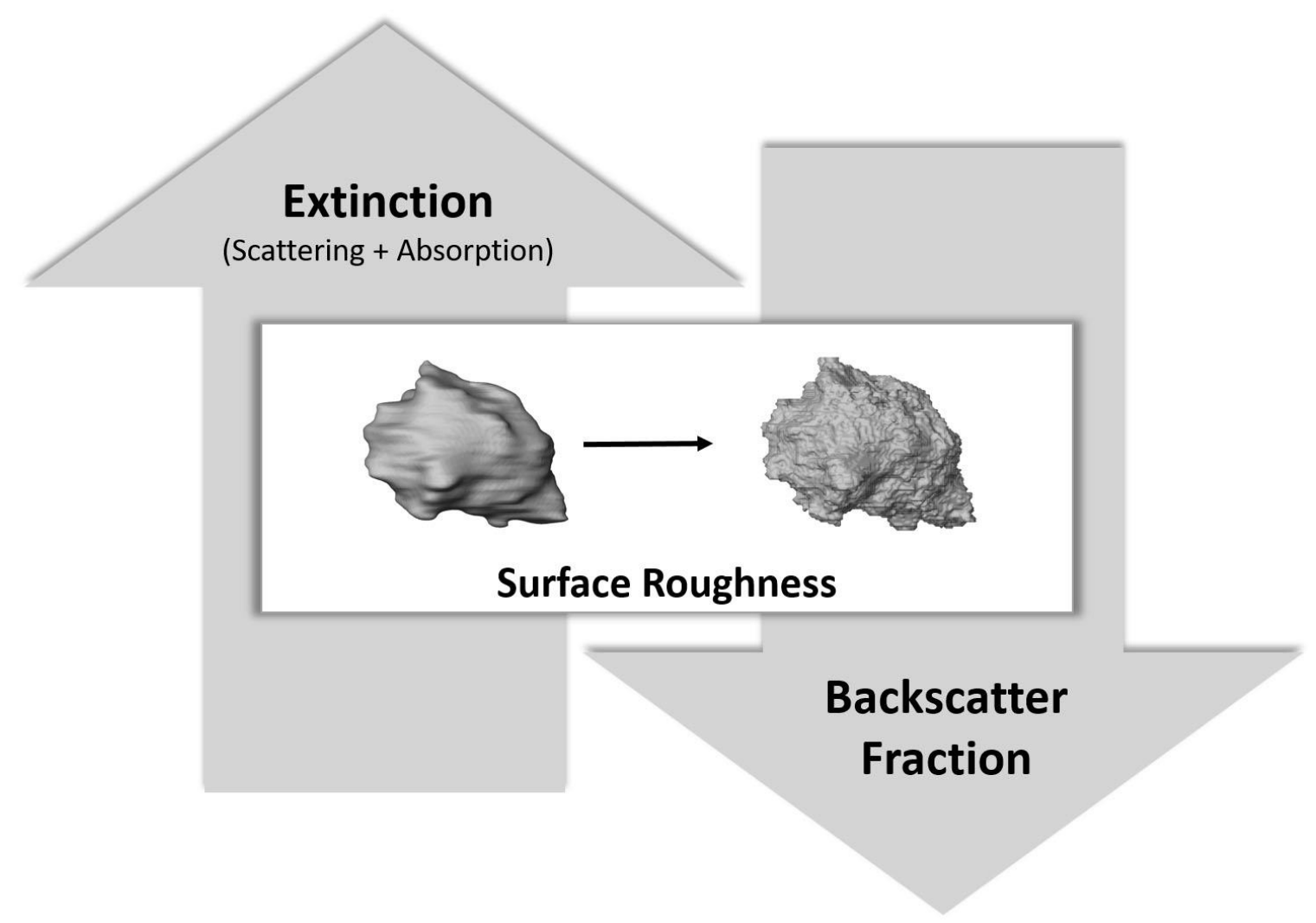

Figure 2. Schematic of the trends observed with an increase in surface roughness. Left arrow represents an increase in particle light extinction, which equals the sum of light scattering and absorption. The right arrow represents a decrease in the backscatter fraction, which is the fraction of light intensity scattered from $90^{\circ}$ to $180^{\circ}$. The representing dust particle was collected at Hawaii's Mauna Loa Observatory.

\section{References}

[1] Kalashnikova, O.V., and Sokolik I.N. (2004). Modeling the radiative properties of nonspherical soilderived mineral aerosols. Journal of Quantitative Spectroscopy and Radiative Transfer, 87, 137- 166.

[2] Yi B., Hsu, C.N., Yang, P., and Tsay, S.C. (2011). Radiative transfer simulation of dust-like aerosols: Uncertainties from particle shape and refractive index. Journal of Aerosol Science, 42, 631-644.

[3] $5^{\text {th }}$ Intergovernmental Panel on Climate Change (IPCC) Report on Climate Change. Chapter 8: Anthropogenic and Natural Radiative Forcing, (2013).

[4] Commercial products identified here specify the means of experimentation. Such identification is not intended to imply recommendation or endorsement by NIST nor that the identified products are necessarily the best available for the purpose.

[5] Draine, B.T., and Flatau, P.J. (1994). Discrete-dipole approximation for scattering calculations. Journal of the Optical Society of America, 11, 1491- 1499. [Source of the code: https://code.google.com/p/ddscat/] [6] Conny, J.M., and Ortiz-Montalvo, D.L. (2017). Effect of heterogeneity and shape on optical properties of urban dust based on three-dimensional modeling of individual particles. Journal of Geophysical Research-Atmospheres, 122, 9816- 9842.

[7] Conny, J.M., Willis, R.D., and Ortiz-Montalvo D.L. (2019). Analysis and optical modeling of individual heterogeneous Asian dust particles collected at Mauna Loa Observatory. Journal of Geophysical Research-Atmospheres, 124, 2702- 2723. 
[8] Conny, J.M., Willis, R.D., and Ortiz-Montalvo D.L. (2020). Optical modeling of single Asian dust and marine air particles: A comparison with geometric particle shapes for remote sensing. Submitted to Journal of Quantitative Spectroscopy and Radiative Transfer. 